\title{
Multiple Layered Solutions of the Nonlocal Bistable Equation
}

\author{
Adam Chmaj * \\ Department of Mathematics \\ Brigham Young University \\ Provo, UT 84602 \\ Xiaofeng Ren ${ }^{\dagger}$ \\ Department of Mathematics and Statistics \\ Utah State University \\ Logan, UT 84322
}

May, 2000

\begin{abstract}
The nonlocal bistable equation is a model proposed recently to study materials whose constitutive relations among the variables that describe their states are nonlocal. It resembles the local bistable equation (the Allen-Cahn equation) in some way, but contains a much richer set of solutions. In this paper we consider two types of solutions. The first are the periodic solutions on a finite interval. These solutions are observed in materials like elastic crystals undergoing martensitic phase transitions and diblock copolymers at low temperatures. They are constructed by a variational method known as the $\Gamma$-limit technique. The second are solutions on the entire real line with transition layers, which are found by the formal matched asymptotics argument. We construct them to compare with the single layer heteroclinic and traveling wave solutions of the local bistable equation. The existence of multiple layered solutions depends on a unique nonlocal feature: the presence of two properly balanced competing effects of the constitutive relation, the oscillation inhibiting effect and the oscillation forcing effect, which coexist at two different length scales.
\end{abstract}

1991 Mathematics Subject Classification: 45G10, 34D15, 49J45.

Keywords: Nonlocality, $\Gamma$-convergence, Matched asymptotics.

Corresponding author: Xiaofeng Ren, Department of Mathematics and Statistics, Utah State University, Logan, UT 84322-3900, USA. Tel: (435) 797-0755, Fax: (435) 797-1822, E-mail: ren@math.usu.edu.

\footnotetext{
* Supported in part by NSF grant DMS-9975451.

${ }^{\dagger}$ Supported in part by NSF grant DMS-9703727.
} 


\section{Introduction}

Among the variables that describe the states of solids, fluids and gases, the classical theory of continuum mechanics assumes local constitutive relations. One variable at a place and a time depends on the other variables and/or their derivatives at the same place and the same time. Examples of such relations are pointwise linear (or nonlinear) strain-stress relations of elastic solids and pressure-density-temperature relations of the ideal, Abel and van der Walls gases.

This classical theory is however inadequate when more complex materials are studied. We mention two such materials in this paper. The first is an elastic crystal that undergoes martensitic phase transitions. The typical equilibrium configuration of this crystal exhibits a characteristic phase mixture of microstructures: common observations demonstrate periodic patterns of piecewise homogeneous elastic domains, each of which has its particular atom formation. The second is a diblock copolymer. In a diblock copolymer, a linear-chain molecule consists of two sub-chains grafted covalently to each other. The subchains are made of two different monomer units. The different sub-chains tend to segregate below some critical temperature, but as they are chemically bonded, only local micro-phase separation occurs.

Recently the use of nonlocal constitutive relations has appeared in the study of such materials. We mention the work of Ren and Truskinovsky [23] which deals with elastic crystals and the work of Ohta and Kawasaki [22] which deals with diblock copolymers.

We will focus on the mathematical aspects of these models and study a prototype that is simple enough so only two variables, one of them being the free energy density and the other being a general phase field, are treated, yet still powerful enough to allow a nonlocal constitutive relation between them, which leads to complex stable configurations of the phase field. When this prototype is linked to the elastic crystal undergoing martensitic transitions, we may view the general phase field as the strain field, and when this prototype is linked to the diblock copolymer, the phase field is the relative concentration of one of the two monomers.

We will let the constitutive relation have two competing effects: one inhibits oscillation and one forces oscillation of the phase field. We will show that when these two effects are properly balanced at two different length scales, the material exhibits periodic structures, as observed in martensites and copolymers.

Now let us denote the energy density of the continuum by $e(x)$. For solids this $x$ is usually the Lagrange coordinate and for gases and fluids the Euler coordinate. $x$ is defined in a domain $\Omega$. Then the total free energy is

$$
I=\int_{\Omega} e(x) d x
$$

The second variable we study is $u(x)$, a general phase field variable. The configuration of the material is described by this variable. Here the nonlocal 
constitutive relation between $e$ and $u$ comes in. However we first look at the dependence of the total free energy on $u$ :

$$
I(u)=\frac{1}{4} \int_{\Omega} \int_{\Omega} J(x, y)(u(x)-u(y))^{2} d x d y+\int_{\Omega} W(u(x)) d x .
$$

The second term on the right side sums over part of the energy density, $W(u)$. This part of the energy density is pointwise and the corresponding energy rises locally. The other term contains the part of the energy arising nonlocally through the self interaction of $u$. In this part $J$ is a function of two variables $x$ and $y$, which measures the self interaction of the field $u$ between the two points $x$ and $y$. This is an assumption on the nonlocal feature of the interaction energy. To see this nonlocality in the constitutive relation between $u$ and $e$, we multiply out the square and rewrite (1.2) as

$$
I(u)=\int_{\Omega}\left[-\frac{1}{2} J[u] u+\frac{j}{2} u^{2}+W(u)\right] d x
$$

where

$$
J[u](x)=\int_{\Omega} J(x, y) u(y) d y, \quad j(x)=\int_{\Omega} J(x, y) d y .
$$

Mathematically $J$ behaves as a kernel.

Comparing (1.1) and (1.3) we see the constitutive relation

$$
e=-\frac{1}{2} J[u] u+\frac{j}{2} u^{2}+W(u)
$$

The total energy (1.2) (and the following (1.5)) can be derived from a microscopic argument. The reader may find the details of such an argument in Bates and Chmaj [3, 4]. Here we summarize their approach. Consider a binary material, whose lattice sites are occupied by blocks, each consisting of A and B atoms. To a site with a block of atoms A (B) only, we assign the spin $+1(-1)$. Let $v(r)$ be the probability that the spin at site $r$ is +1 . Then the expected value of this spin is $u(r)=2 v-1 \in[-1,1]$.

The Helmholtz free energy of our system is given by

$$
E=H-T S,
$$

where $H$ is the internal interaction energy, $T$ the absolute temperature and $S$ the entropy.

Similarly to the Ising model (see [26]), the internal energy has the form

$$
\begin{aligned}
H(u)=- & \frac{1}{4} \sum_{r, r^{\prime}}\left[J^{A A}\left(r, r^{\prime}\right)(1+u(r))\left(1+u\left(r^{\prime}\right)\right)+J^{B B}\left(r, r^{\prime}\right)(1-u(r))\left(1-u\left(r^{\prime}\right)\right)\right. \\
& \left.+J^{A B}\left(r, r^{\prime}\right)\left((1+u(r))\left(1-u\left(r^{\prime}\right)\right)+(1-u(r))\left(1+u\left(r^{\prime}\right)\right)\right)\right],
\end{aligned}
$$

where $J^{A A}\left(r, r^{\prime}\right), J^{B B}\left(r, r^{\prime}\right), J^{A B}\left(r, r^{\prime}\right)$ measure the interaction energies between sites $r$ and $r^{\prime}$ with spins 1 and $1,-1$ and $-1,1$ and -1 , which we assume 
are non-negative, symmetric and translationally invariant in $\left(r, r^{\prime}\right)$. This means that $J^{A A, B B, A B}(r, r)$ depends on $\left|r-r^{\prime}\right|$ only.

In the Bragg-Williams [9] approximation, the total entropy has the form

$$
S(u)=-K \sum_{r}[(1+u(r)) \log (1+u(r))+(1-u(r)) \log (1-u(r))],
$$

where $K>0$. Rearranging (1.6), we obtain

$$
\begin{aligned}
E(u)= & \frac{1}{4} \sum_{r, r^{\prime}}\left(\frac{1}{2} J^{A A}\left(r, r^{\prime}\right)+\frac{1}{2} J^{B B}\left(r, r^{\prime}\right)-J^{A B}\left(r, r^{\prime}\right)\right)\left(u(r)-u\left(r^{\prime}\right)\right)^{2}-\frac{1}{2} k_{1} \\
& -\sum_{r}\left\{\frac{1}{2} k_{2}(r) u(r)-\frac{1}{2} k_{3}(r) u^{2}(r)\right. \\
& +T K[(1+u(r)) \log (1+u(r))+(1-u(r)) \log (1-u(r))]\},
\end{aligned}
$$

where

$$
\begin{aligned}
k_{1} & =\sum_{r, r^{\prime}}\left(\frac{1}{2} J^{A A}\left(r, r^{\prime}\right)+\frac{1}{2} J^{B B}\left(r, r^{\prime}\right)+J^{A B}\left(r, r^{\prime}\right)\right), \\
k_{2}(r) & =\sum_{r^{\prime}}\left(J^{A A}\left(r, r^{\prime}\right)-J^{B B}\left(r, r^{\prime}\right)\right), \\
k_{3}(r) & =\sum_{r^{\prime}}\left(\frac{1}{2} J^{A A}\left(r, r^{\prime}\right)+\frac{1}{2} J^{B B}\left(r, r^{\prime}\right)-J^{A B}\right)\left(r, r^{\prime}\right) .
\end{aligned}
$$

We drop $k_{1}$ in (1.7) since it only affects the energy of $u$ by a constant, and rewrite (1.7) as

$$
E(u)=\frac{1}{4} \sum_{r, r^{\prime}} J\left(r, r^{\prime}\right)\left(u(r)-u\left(r^{\prime}\right)\right)^{2}-\sum_{r} W(u(r), r),
$$

where

$$
\begin{aligned}
J\left(r, r^{\prime}\right)= & \frac{1}{2} J^{A A}\left(r, r^{\prime}\right)+\frac{1}{2} J^{B B}\left(r, r^{\prime}\right)-J^{A B}\left(r, r^{\prime}\right), \\
W(u, r)= & \frac{1}{2} k_{2}(r) u-\frac{1}{2} k_{3}(r) u^{2} \\
& +T K[(1+u) \log (1+u)+(1-u) \log (1-u)] .
\end{aligned}
$$

$J\left(r, r^{\prime}\right)$ may change sign, since $J^{A A}\left(r, r^{\prime}\right), J^{B B}\left(r, r^{\prime}\right)$ and $J^{A B}\left(r, r^{\prime}\right)$ are all non-negative. When the lattice is infinite, the translational invariance of $J^{A A}\left(r, r^{\prime}\right), J^{B B}\left(r, r^{\prime}\right)$ and $J^{A B}\left(r, r^{\prime}\right)\left(J^{A A, B B, A B}\left(r, r^{\prime}\right)=J^{A A, B B, A B}\left(r-r^{\prime}\right)\right)$ implies that the quantities $k_{2}$ and $k_{3}$ are independent of $r$. In this case $W(u, r)=$ $W(u)$. In this paper we either assume that the lattice is infinite as in Section 3 , or assume, as in Section 2, that the lattice is bounded but the $J^{A A, B B, A B}$ 's are 'almost' translationally invariant. So we make the assumption $W(u, r)=W(u)$. 
We find (1.2) to be the continuous version of (1.8). When $\Omega$ is bounded, $J$ may incorporate interactions between sites and the boundary, so $J$ is not translationally invariant. We only assume that $J(x, y)=J(y, x)$. When $\Omega$ is unbounded, (1.2) is understood in a formal sense. In this case $J$ is necessarily translationally invariant: $J(x, y)=J(|x-y|)$. In either case according to (1.9) $J$ may change sign.

Two remarks need to be made at this point about $W$. First, $j(x)$ is simply the continuous version of $k_{3}(r)$. Then $\frac{j}{2} u^{2}+W(u)$ 's discrete counterpart is

$$
\frac{1}{2} k_{2} u+T K[(1+u) \log (1+u)+(1-u) \log (1-u)]
$$

which is convex in $u \in(-1,1)$. Surprisingly the convexity of $\frac{j}{2} u^{2}+W(u)$ in $u$ is also a key mathematical assumption in this paper. In Section 2 it guarantees the well-posedness of the variational problem and in Section 3 it gives the first inner expansion term.

Second, from (1.10) we see that only when the temperature is low, i.e., $T$ is small compared to $k_{3}, W$ is non-convex in $u \in(-1,1)$. Recall that for many binary materials microphases separate only at low temperatures. In this paper we only study this non-convex case of $W$. We do not restrict ourselves to the specific form (1.10), but after rescaling we assume that $W$ is a double well function with two local minima at -1 and 1 . Because of the presence of $k_{2}$ in (1.10), the two wells of $W$ are not necessarily balanced, i.e., $W(-1)$ is not in general equal to $W(1)$.

Since the configuration of the material is reflected in $u$, we look for stable $u$ 's. In Section 2 we take an energy approach to find such $u$ 's. We treat $I$ in (1.2) as a functional defined on phase fields $u$. Then the stable $u$ 's are defined mathematically to be the local minimizers of $I$. They satisfy the Euler-Lagrange equation of (1.2):

$$
-J[u]+j u+f(u)=0,
$$

where $f(u)=W^{\prime}(u)$. Our study of minimizers there is rigorous and is based on the mathematical theory of $\Gamma$-convergence.

As motivated by martensitic crystals and diblock copolymers, we are interested in periodic solutions on a finite interval $(0,1)$. We will show that such solutions exist when the kernel $J$ has a particular form:

$$
J(x, y)=\frac{1}{\epsilon} J^{s}\left(\frac{x-y}{\epsilon}\right)-\epsilon J^{l}(x, y)
$$

where both $J^{s}$ and $J^{l}$ are non-negative and $\epsilon$ is small. This form of $J$ gives two competing effects. The first is a oscillation inhibiting effect that penalizes spatial inhomogeneity of $u$, mathematically reflected in the first part of the right side of the above expression. This effect is localized and acts on a scale of $\epsilon$. The second effect forces oscillation of $u$, as reflected in the second part of the expression. It acts weakly, of order $\epsilon$, on a large scale, comparable to the size of the material sample. Our results in Section 2 show that this particular balance 
of the competing effects gives local energy minimizers $u$ that are periodic in $x$ and the length scale of the periods is comparable to that of the sample size.

In Section 3 we examine some other mathematical properties of (1.2). We take a formal, matched asymptotics approach to consider the negative gradient flow of (1.2), which is a simple dynamic law governing the motion of the field $u$. It has the form

$$
u_{t}=J[u]-j u-f(u) .
$$

We now make a comparison with the local bistable equation, the AllenCahn equation. Consider (1.2) with $\Omega=R$ and $J$ translationally invariant. Change variables using $\eta=\frac{x-y}{2}, \xi=\frac{x+y}{2}$, and expand $u(x)=u(\xi+\eta)$ and $u(y)=u(\xi-\eta)$ about $\xi$, to obtain the formal expression

$$
\begin{gathered}
\frac{1}{2} \int_{R} \int_{R} J(2 \eta)\left(\sum_{k=0}^{\infty} \frac{D^{2 k+1} u(\xi) \eta^{2 k+1}}{(2 k+1) !}\right)^{2} d \xi d \eta+\int_{R} W(u(x)) d x \\
\simeq \int_{R}\left(c_{1}\left|u^{\prime}(\xi)\right|^{2}-c_{2}\left|u^{\prime \prime}(\xi)\right|^{2}+\ldots\right) d \xi+\int_{R} W(u(x)) d x
\end{gathered}
$$

where $c_{1}=\frac{1}{2} \int_{R} J(2 \eta) \eta^{2} d \eta, c_{2}=\frac{1}{12} \int_{R} J(2 \eta) \eta^{4} d \eta$, etc. Truncating the summation in (1.14) at the first term gives the energy

$$
\int_{R}\left[c_{1}\left|u^{\prime}(x)\right|^{2}+W(u(x))\right] d x
$$

whose $L^{2}$-gradient flow is the Allen-Cahn equation

$$
u_{t}=c_{1} u_{x x}-f(u) .
$$

Note that for this equation to be well posed, we need to assume that $c_{1}>0$. Truncating the summation in (1.14) at the second term and assuming that $c_{2}<0$ gives an energy functional very similar to the one which appears in the Swift-Hohenberg model [25], as well as the theory of Lifshitz points in condensed matter systems [19]. Note that $c_{2}<0$ is only possible when $J$ changes sign.

From a constitutive relation viewpoint, expressions like (1.15) imply a local relation between $e$ and $u$ like $e=c_{1}\left|u^{\prime}\right|^{2}+W(u)$. In essence they assume that $e(x)$, the energy density at $x$, depends on $u(x), u^{\prime}(x), \ldots$, property of $u$ at the infinitesimal neighborhood of $x$. They are therefore considered as higher order local models.

The equation (1.16) has monotone heteroclinic orbits when $f$ is balanced, i.e. , $\int_{-1}^{1} f=W(1)-W(-1)=0$. When $f$ is not balanced, i.e., $\int_{-1}^{1} f=$ $W(1)-W(-1) \neq 0,(1.16)$ has monotone traveling wave solutions.

In Section 3 we look for stable solutions of (1.13) on the entire line that are of the homoclinic, heteroclinic, and traveling wave types. These solutions are not necessarily monotone.

These solutions in general have higher average energy than the periodic solutions, found in Section 2, do. We conjecture that they are only visible as 
material configurations during early stages of evolution. As the temperature cools they should give in to more periodic configurations.

On the entire line, the kernel $J$ takes a similar but translationally invariant form:

$$
J(r)=\frac{1}{\epsilon} J^{s}\left(\frac{r}{\epsilon}\right)-\epsilon J^{l}(r), r=|x-y| .
$$

This again separates two scales, $\epsilon$ and 1 . However in this section we only assume $J^{s}$ to be non-negative. $J^{l}$ may change sign, so $J$ may change sign more than once.

We use a formal matched asymptotics approach in this section. We look for stationary solutions of (1.13), formally stable in the sense that the linearized operators have no unstable eigenvalues. Barring degenerate cases, the notion of stability in the sense of Section 2 is consistent with the notion of stability in Section 3.

Higher order truncations like (1.14) in the case $J \geq 0$ were studied in in [7], [8] and [5]. Equation (1.13) was proposed recently in [6]. It was subsequently studied in several papers, e.g., [20], [11], [12], [3] and [18]. A discrete version of (1.13) was proposed and studied in [4]. Most of these results address the case $J \geq 0$, for which the equation (1.13) has a comparison principle. In particular, it was observed in [6], [11], [12] and [3] that in contrast to the Allen-Cahn equation, (1.11) admits discontinuous solutions. The case when $J$ changes sign seems to be considerably more difficult, mainly due to the lack of the comparison principle for (1.13). To our knowledge, it was studied only in [3] and [18]. In particular, in [3] it is shown that for $j u+f(u)$ non-monotone and $j$ sufficiently small, there exist both asymptotically stable and unstable solutions of (1.11) having discontinuities across arbitrarily prescribed interfaces.

\section{The $\Gamma$-convergence approach}

We now turn our attention to bounded intervals. Consider (1.11) with $\Omega=$ $(0,1)$. We separate the long range effect of $J$ and the short range effect as in (3.1), and assume that the short range effect of $J$ is localized and the long range effect is small. More precisely

$$
J(x, y)=J_{\epsilon}^{s}-\epsilon J^{l}=\frac{1}{\epsilon} J^{s}\left(\frac{x-y}{\epsilon}\right)-\epsilon J^{l}(x, y) .
$$

The conditions on $J^{s}$ and $J^{l}$ are

H1 The short range effect is translationally invariant, even and penalizes oscillation, i.e. $J^{s}(-r)=J^{s}(r) \geq 0, \int_{R} J^{s}(r) d r>0$.

H2 On the growth of $J^{s}$ we assume $\int_{R} J^{s}(r) d r<\infty, \int_{R}|r| J^{s}(r) d r<\infty$.

H3 $J^{l}$ is symmetric, i.e. $J^{l}(x, y)=J^{l}(y, x)$. The function $j^{l}(x)=\int_{0}^{1} J^{l}(x, y) d y$ is in $L^{\infty}(0,1)$ and the operator $J^{l}: u \rightarrow J^{l}[u]$ is bounded from $L^{2}(0,1)$ to itself. 

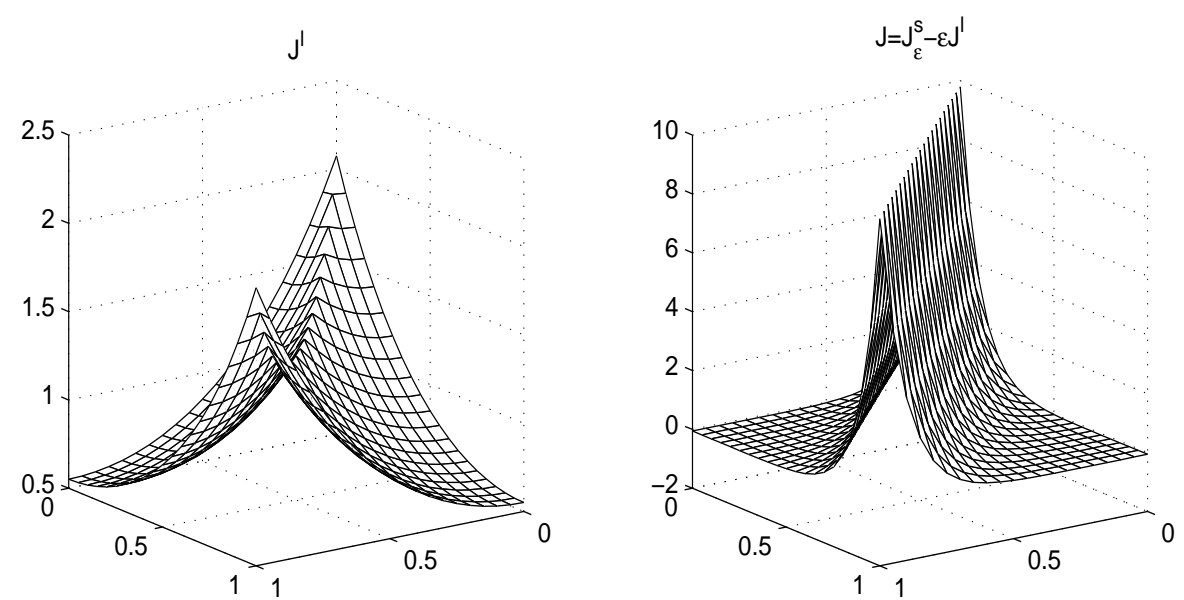

Figure 1: An example of $J^{l}$ and $J=J_{\epsilon}^{s}-\epsilon J^{l}$.

H4 The operator $J: u \rightarrow J[u]$ is compact from $L^{2}(0,1)$ to itself, which is satisfied if, e.g., $J \in L^{2}((0,1) \times(0,1))$.

Figure 2 shows an example of $J$. The first plot is the picture of $J^{l}>0$, which is given later in (2.11), (with the parameter $\gamma=0.5$ ). The second plot is the picture of $J$ with $J^{s}(r)=\exp (-|r|)>0$ and $\epsilon=0.1$. This function $J$ changes sign. It is positive when $(x, y)$ is close to the diagonal $x=y$ and negative elsewhere, although the negative part of $J$ is not easy to recognize in the picture since $\epsilon$ is small.

The local term $f$ is continuous and allowed to have an expansion in $\epsilon, f=$ $f_{0}+\epsilon f_{1}$. Let $W(s)=\int_{-1}^{s} f(r) d r$. In terms of $\epsilon, W=W_{0}+\epsilon W_{1}$, where $W_{0}(s)=$ $\int_{-1}^{s} f_{0}(r) d r$ and $W_{1}(s)=\int_{-1}^{s} f_{1}(r) d r$. The conditions on $f$ are imposed on $W$.

H5 $W_{0}$ is a double well function of equal depth. So $W_{0}(s) \geq 0$ for all $s \in R$, and only when $s= \pm 1, W_{0}(s)=0$, i.e. $\int_{-1}^{1} f_{0}(r) d r=0$.

H6 The growth rate of $W_{0}$ is at least quadratic, i.e. there exists $C>0$ such that $W_{0}(s) \geq C s^{2}-C$ for all $s \in R$.

H7 The growth rate of $W_{1}$ is at most quadratic, i.e. there exists $C>0$ such that $\left|W_{1}(s)\right| \leq C|s|^{2}+C$ for all $s \in R$.

H8 For small $\epsilon, \frac{j(x) s^{2}}{2}+W(s)$ is convex in $s$ for all $x$.

Condition H8, mentioned in Section 1, is very important. We will see an analogy, $j^{s}+f_{0}^{\prime}>0$, in Section 3. It implies that $j u+f(u)$ is continuously monotone in $u$. Therefore any $L^{2}$ solution $u$ of (1.11) is continuous if $J$ maps $L^{2}$-functions to continuous functions. Since $W$ is not convex, $\mathbf{H} 8$ requires that $j$ is positive and large. 
We seek solutions of (1.11) by minimizing the functional $I_{\epsilon}=I$ defined in (1.2). In terms of $\epsilon$ we set $I_{\epsilon}=I_{\epsilon}^{s}+\epsilon I^{l}$, where

$$
\begin{aligned}
I_{\epsilon}^{s}(u) & =\frac{1}{4} \int_{0}^{1} \int_{0}^{1} J_{\epsilon}^{s}(x, y)(u(x)-u(y))^{2} d x d y+\int_{0}^{1} W_{0}(u) d x \\
I^{l}(u) & =-\frac{1}{4} \int_{0}^{1} \int_{0}^{1} J^{l}(x, y)(u(x)-u(y))^{2} d x d y+\int_{0}^{1} W_{1}(u) d x \\
& =\int_{0}^{1}\left[\frac{1}{2} J^{l}[u] u-\frac{j^{l} u^{2}}{2}+W_{1}(u)\right] d x .
\end{aligned}
$$

The admissible set of $I_{\epsilon}$ is taken to be $L^{2}(0,1)$, even though for some $u \in L^{2}$, $I_{\epsilon}^{s}(u)$ can be $\infty$. $I^{l}(u)$ is always finite, so $I_{\epsilon}: L^{2} \rightarrow(-\infty, \infty]$. The next proposition collects some basic properties of $I_{\epsilon}$.

Proposition 2.1 1. There exists a constant $c$ independent of small $\epsilon$ so that for every $u \in L^{2}(0,1), I_{\epsilon}(u) \geq c \epsilon$.

2. There exists $C>0$, independent of small $\epsilon$, so that $\|u\|_{2} \leq C I_{\epsilon}(u)+C$.

3. For small $\epsilon, I_{\epsilon}$ is weakly lower semi-continuous on $L^{2}(0,1)$.

4. $I_{\epsilon}$ has a global minimum if $\epsilon$ is small.

5. On any convex $X \subset L^{2}(0,1)$, for small $\epsilon$ there exists $u_{X} \in \bar{X}$ such that $I_{\epsilon}\left(u_{X}\right)=\inf _{u \in X} I_{\epsilon}(u)$.

Proof. 1 and 2 follow easily from H3, H6 and H7. For 3 we rewrite $I_{\epsilon}(u)$ as $\int_{0}^{1}\left[\frac{j^{2} u^{2}}{2}+W(u)\right] d x-\frac{1}{2} \int_{0}^{1} J[u] u d x$. We see that the first functional is convex by $\mathbf{H} \mathbf{8}$ and the second compact. Then $I_{\epsilon}$ is weakly lower semi-continuous. 4 and 5 follow from 1-3.

It turns out that $\epsilon^{-1} I_{\epsilon}$ has a well defined singular limit $K+I^{l}$. This limit is defined on $L^{2}(0,1)$, though only for $u \in B V((0,1),\{-1,1\}),\left(K+I^{l}\right)(u)$ is finite. Here $B V((0,1),\{-1,1\})$ is the space of functions of finite variation with the range $\{-1,1\}$. Every function there changes its value between -1 and 1 finitely many times. The formal definition of $K+I^{l}$ is

$$
\left(K+I^{l}\right)(u)=\left\{\begin{array}{ll}
c_{0} \frac{\|D u\|(0,1)}{2}+I^{l}(u) & \text { if } u \in B V((0,1),\{-1,1\}) \\
\infty & \text { otherwise }
\end{array} .\right.
$$

Here $K(u)=c_{0} \frac{\|D u\|(0,1)}{2}$ is equal to the number of jumps $u$ has multiplied by a constant $c_{0}$. This number of jumps is mathematically equal to half the size of $(0,1)$ under the measure $|D u|$. The constant $c_{0}$ depends on the solution of

$$
-J^{s} * U_{0}^{+}+\left(\int_{R} J^{s}(r) d r\right) U_{0}^{+}+f_{0}\left(U_{0}^{+}\right)=0 \text { in } R, U_{0}^{+}( \pm \infty)= \pm 1 .
$$

The existence and uniqueness, up to translation, of such a solution is shown in [6]. Then $c_{0}$ is a positive number equal to

$$
\frac{1}{4} \int_{R} \int_{R} J^{s}(y, r)\left(U_{0}^{+}(y)-U_{0}^{+}(r)\right)^{2} d y d r+\int_{R} W_{0}\left(U_{0}^{+}(r)\right) d r .
$$


Proposition $2.2 \epsilon^{-1} I_{\epsilon} \Gamma$-converges to $K+I^{l}$ as $\epsilon \rightarrow 0$ in the following sense.

1. For every $\left\{u_{\epsilon}\right\} \subset L^{2}(0,1)$ with $\lim _{\epsilon \rightarrow 0} u_{\epsilon}=u, \liminf _{\epsilon \rightarrow 0} \epsilon^{-1} I_{\epsilon}\left(u_{\epsilon}\right) \geq$ $\left(K+I^{l}\right)(u)$

2. For every $u \in L^{2}(0,1) \cap B V((0,1),\{-1,1\})$, there exists a family $\left\{u_{\epsilon}\right\} \subset$ $L^{2}(0,1)$ such that $\lim _{\epsilon \rightarrow 0} u_{\epsilon}=u$, and $\lim _{\sup _{\epsilon \rightarrow 0}} \epsilon^{-1} I_{\epsilon}\left(u_{\epsilon}\right) \leq\left(K+I^{l}\right)(u)$.

Proof. It was proved by Alberti and Bellettini in [1], Theorem 1.1 (ii) and (iii), that $\epsilon^{-1} I_{\epsilon}^{s} \Gamma$-converges to $K$, where $\epsilon^{-1} I_{\epsilon}^{s}$ and $K$ are defined on $L^{1}$. After a small modification of their proof, we find that $\epsilon^{-1} I_{\epsilon}^{s} \Gamma$-converges to $K$ in $L^{2}(0,1)$. The $I^{l}$ term is a $L^{2}$-continuous perturbation of $\epsilon^{-1} I_{\epsilon}^{s}$. From the definition of $\Gamma$-convergence, we deduce that $\epsilon^{-1} I_{\epsilon} \Gamma$-converges to $K+I^{l}$.

We would like to point out that this property, and that of Proposition 2.3, of $I_{\epsilon}$ does not require the conditions $\mathbf{H} 4$ and H8. Those two conditions are mainly used for the existence of minima of $I_{\epsilon}$.

Proposition 2.3 Let $\epsilon_{n}$ be a sequence of positive numbers converging to 0 , and $\left\{u_{n}\right\}$ a sequence in $L^{2}(0,1)$. If $\epsilon_{n}^{-1} I_{\epsilon_{n}}\left(u_{n}\right)$ is bounded above in $n$, then $\left\{u_{n}\right\}$ is relatively compact in $L^{2}(0,1)$ and its cluster points belong to $B V((0,1),\{-1,1\})$.

Proof. Because of $\mathbf{H 3}, \mathbf{H} \mathbf{6}$ and $\mathbf{H 7}, \epsilon_{n}^{-1} I_{\epsilon_{n}}^{s}\left(u_{n}\right)$ is bounded above. Then [1] Theorem 1.1 (i), after a change from $L^{1}$ to $L^{2}$, implies that $\left\{u_{n}\right\}$ is relatively compact in $L^{2}(0,1)$ and its cluster points belong to $B V((0,1),\{-1,1\})$.

A useful property following Propositions 2.2 and 2.3 is that isolated local minima of the $\Gamma$-limit persist under small perturbation. Denote an open ball in $L^{2}(0,1)$ centered at $u_{0}$ of radius $r$ by $B_{r}\left(u_{0}\right)$.

Proposition 2.4 Let $\delta>0$ and $u_{0} \in L^{2}(0,1)$ be such that $\left(K+I^{l}\right)\left(u_{0}\right)<$ $\left(K+I^{l}\right)(u)$ for all $u \in B_{\delta}\left(u_{0}\right)$ with $u \neq u_{0}$. Then for small $\epsilon$ there exists $u_{\epsilon} \in B_{\delta / 2}\left(u_{0}\right)$ with $I_{\epsilon}\left(u_{\epsilon}\right) \leq I_{\epsilon}(u)$ for all $u \in B_{\delta / 2}\left(u_{0}\right)$. In addition $\lim _{\epsilon \rightarrow 0} \| u_{\epsilon}-$ $u_{0} \|_{2}=0$.

Proof. Follow, for example, the proof of [24] Proposition 2.3, [21], or [13], with the help of Proposition 2.1.

Concerning the global minima of $I_{\epsilon}$, the following is an easy consequence of Propositions 2.1, 2.2 and 2.3.

Proposition 2.5 Let $\left\{\epsilon_{n}\right\}$ be a positive sequence converging to 0 , and $u_{\epsilon_{n}}$ be a global minimum of $I_{\epsilon_{n}}$ in $L^{2}(0,1)$. Then $\left\{u_{\epsilon_{n}}\right\}$ is relatively compact in $L^{2}(0,1)$ and each of its cluster points is a global minimum of $K+I^{l}$.

Based on propositions 2.4 and 2.5, we look for local and global minima of $K+I^{l}$. The structure of $B V((0,1),\{-1,1\})$ is rather simple. For each integer $N \geq 0$, we have a subset

$$
A_{N}=\left\{u \in B V((0,1),\{-1,1\}): \frac{\|D u\|(0,1)}{2}=N\right\},
$$


the set of functions with $N$ jumps. This set can be further divided into $A_{N}^{-1}$ and $A_{N}^{1}$.

$$
\begin{array}{r}
A_{N}^{-1}=\left\{u \in A_{N}: u(x)=-1, \text { if } x \text { is between } 0\right. \\
\text { and the first jump point of } u\}
\end{array}
$$

Note $A_{0}^{-1}=\{u \equiv-1\}$ and $A_{0}^{1}=\{u \equiv 1\}$. Now we have a mutually disjoint decomposition

$$
B V((0,1),\{-1,1\})=\cup_{N=0}^{\infty}\left(A_{N}^{-1} \cup A_{N}^{1}\right) .
$$

We can now minimize $K+I^{l}$ in each $A_{N}^{ \pm 1}$. In $A_{0}^{-1},\left(K+I^{l}\right)(u \equiv-1)=$ $W_{1}(-1)$, and in $A_{0}^{1},\left(K+I^{l}\right)(u \equiv 1)=W_{1}(1)$. Every $u \in A_{N}^{ \pm 1}, N \geq 1$, is identified by its jump points, $\xi_{1}, \xi_{2}, \ldots, \xi_{N}$. Then $K+I^{l}=c_{0} N+I^{l}(u)$ can be expressed as a function of $\xi_{i}$. The partial derivatives of this function can be calculated using (2.3). In $A_{N}^{-1}$,

$$
\begin{aligned}
\frac{\partial I^{l}(u)}{\partial \xi_{1}}= & \frac{\partial}{\partial \xi_{1}}\left[-\frac{1}{2} \int_{0}^{\xi_{1}} J^{l}[u](x) d x+\frac{1}{2} \int_{\xi_{1}}^{\xi_{2}} J^{l}[u](x) d x+\ldots\right. \\
& \left.+\xi_{1} W_{1}(-1)+\left(\xi_{2}-\xi_{1}\right) W_{1}(1)+\ldots\right] \\
= & -J^{l}[u]\left(\xi_{1}\right)+\frac{1}{2} \int_{0}^{1} \frac{\partial J^{l}[u]}{\partial \xi_{1}} u(x) d x+W_{1}(-1)-W_{1}(1) \\
= & -J^{l}[u]\left(\xi_{1}\right)-J^{l}[u]\left(\xi_{1}\right)-\int_{-1}^{1} f_{1}(r) d r,
\end{aligned}
$$

since

$$
\frac{\partial J^{l}[u]}{\partial \xi_{1}}=\frac{\partial}{\partial \xi_{1}}\left[-\int_{0}^{\xi_{1}} J^{l}(x, y) d y+\int_{\xi_{1}}^{\xi_{2}} J^{l}(x, y) d y+\ldots\right]=-2 J^{l}\left(x, \xi_{1}\right) .
$$

After a similar computation in $A_{N}^{1}$, we deduce

$$
\frac{\partial I^{l}(u)}{\partial \xi_{i}}=\left\{\begin{array}{rl}
(-1)^{i} 2 J^{l}[u]\left(\xi_{i}\right)+(-1)^{i} \int_{-1}^{1} f_{1}(r) d r, & u \in A_{N}^{-1} \\
-(-1)^{i} 2 J^{l}[u]\left(\xi_{i}\right)-(-1)^{i} \int_{-1}^{1} f_{1}(r) d r, & u \in A_{N}^{1}
\end{array} .\right.
$$

A local minimum (or critical point) of $I^{l}$ in $A_{N}^{ \pm 1}$ is a local minimum (or critical point) of $K+I^{l}$ in $A_{N}^{ \pm 1}$. Such a point satisfies the equation

$$
J^{l}[u]\left(\xi_{i}\right)=-\frac{1}{2} \int_{-1}^{1} f_{1}(r) d r
$$

We will not discuss the solvability of (2.10) for general $J^{l}$ here. Instead we study a particular $J^{l}$. This $J^{l}$ was used by Ren and Truskinovsky [23] to model elastic materials with oscillatory microphases. It is so chosen that the limitation that $u(x)$ can not interact with points outside the domain $(0,1)$ is balanced by 
the fact that $J^{l}(x, y)$ is larger when $x$ and $y$ are closer to the boundary (see Figure 2, plot 1). The expression of $J^{l}$ is

$$
J^{l}(x, y)=\frac{1}{\gamma\left(e^{\frac{1}{\gamma}}-e^{-\frac{1}{\gamma}}\right)}\left[\cosh \left(\frac{x+y-1}{\gamma}\right)+\cosh \left(\frac{|x-y|-1}{\gamma}\right)\right], \gamma>0
$$

which is Green's function of the linear differential equation $-\gamma^{2} v^{\prime \prime}+v=u$, $v^{\prime}(0)=v^{\prime}(1)=0$.

Let $u \in A_{N}^{ \pm 1}$ be a solution of (2.10), identified with $\left(\xi_{1}, \xi_{2}, \ldots, \xi_{N}\right)$ and $v=$ $J^{l}[u]$. Since $v\left(\xi_{1}\right)=v\left(\xi_{2}\right)=-\frac{1}{2} \int_{-1}^{1} f_{1}(r) d r$ by $(2.10), v$ is obtained on $\left(\xi_{1}, \xi_{2}\right)$ by solving $-\gamma^{2} v^{\prime \prime}+v=\mp 1$. In particular $v^{\prime}\left(\xi_{1}\right)=-v^{\prime}\left(\xi_{2}\right)$. Then consider $v$ on $\left(0, \xi_{1}\right)$ and $\left(\xi_{2}, \xi_{3}\right)$, where it satisfies the same linear differential equation with the same right side, \pm 1 . Also because $v\left(\xi_{1}\right)=v\left(\xi_{2}\right)=v\left(\xi_{3}\right), v^{\prime}(0)=0$, and $v^{\prime}\left(\xi_{1}\right)=-v^{\prime}\left(\xi_{2}\right)$, we find that $\xi_{3}-\xi_{2}=2 \xi_{1}$. Repeating this argument over other intervals, we deduce

$$
2 \xi_{1}=\xi_{3}-\xi_{2}=\xi_{5}-\xi_{4}=\ldots, \quad \xi_{2}-\xi_{1}=\xi_{4}-\xi_{3}=\xi_{6}-\xi_{5}=\ldots
$$

which implies $\xi_{1}+\frac{\xi_{2}-\xi_{1}}{2}=\frac{1}{N}$. However unless $\int_{-1}^{1} f_{1}(r) d r=0, \xi_{1} \neq \frac{\xi_{2}-\xi_{1}}{2}$. To find $\xi_{1}$ we write down, in the case $u \in A_{N}^{-1}$,

$$
\begin{aligned}
& v(x)=-1+\frac{1-\frac{1}{2} \int_{-1}^{1} f_{1}(r) d r}{\cosh \left(\frac{\xi_{1}}{\gamma}\right)} \cosh \left(\frac{x}{\gamma}\right), x \in\left(0, \xi_{1}\right), \\
& v(x)=1+\frac{-1-\frac{1}{2} \int_{-1}^{1} f_{1}(r) d r}{\cosh \left(\frac{1}{\gamma N}-\frac{\xi_{1}}{\gamma}\right)} \cosh \left(\frac{1}{\gamma N}-\frac{x}{\gamma}\right), x \in\left(\xi_{1}, \xi_{2}\right) .
\end{aligned}
$$

Since $v$, the solution of a second order ODE, is in $C^{1,1}(0,1), v^{\prime}$ is continuous at $\xi_{1}$. Therefore

$$
\left[1-\frac{1}{2} \int_{-1}^{1} f_{1}(r) d r\right] \tanh \left(\frac{\xi_{1}}{\gamma}\right)=\left[1+\frac{1}{2} \int_{-1}^{1} f_{1}(r) d r\right] \tanh \left(\frac{1}{\gamma N}-\frac{\xi_{1}}{\gamma}\right)
$$

if $u \in A_{N}^{-1}$, or

$$
\left[1+\frac{1}{2} \int_{-1}^{1} f_{1}(r) d r\right] \tanh \left(\frac{\xi_{1}}{\gamma}\right)=\left[1-\frac{1}{2} \int_{-1}^{1} f_{1}(r) d r\right] \tanh \left(\frac{1}{\gamma N}-\frac{\xi_{1}}{\gamma}\right)
$$

if $u \in A_{N}^{1}$. They have solutions $\xi_{1} \in(0,1 / N)$ in terms of $N$ if and only if

$$
-2<\int_{-1}^{1} f_{1}(r) d r<2
$$

Under condition (2.15) we have found in each $A_{N}^{ \pm 1}$ a unique critical point of $I^{l}$. Let us denote them by $\chi_{N}^{ \pm 1}$. The step functions shown in Figure 2 are $\chi_{7}^{ \pm 1}$ and $\chi_{8}^{ \pm 1}$, and the continuous functions are their corresponding $J^{l}\left[\chi^{ \pm 1}\right]$, where $\gamma=0.1, \int_{-1}^{1} f_{1}(r) d r=-0.4$. 

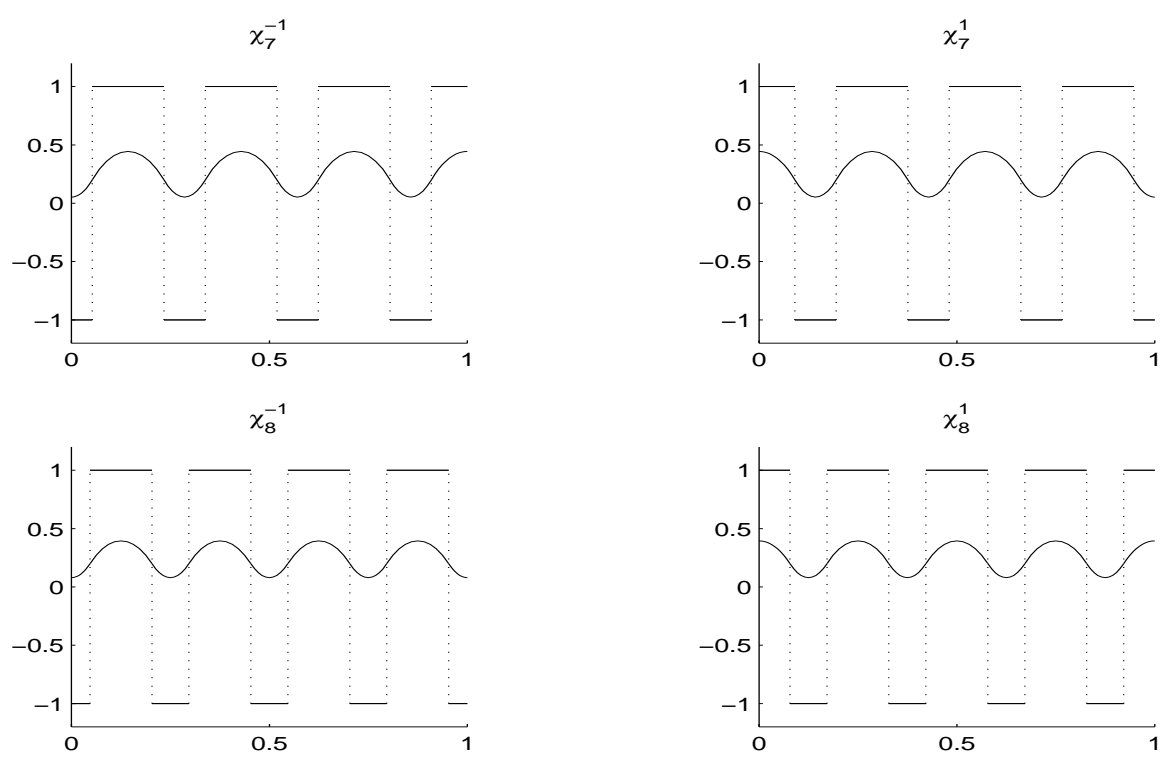

Figure 2: Some $\chi_{N}^{ \pm 1}$ and $J^{l}\left[\chi_{N}^{ \pm 1}\right]$.

In order to determine whether $\chi_{N}^{ \pm 1}$ are local minima of $K+I^{l}$, we compute $I^{l}\left(\chi_{N}^{ \pm 1}\right)$. Here $j^{l}=1$ and $v=J^{l}\left[\chi_{N}^{ \pm 1}\right]$, so

$$
\begin{aligned}
I^{l}\left(\chi_{N}^{-1}\right) & =\int_{0}^{1}\left[\frac{1}{2} \chi_{N}^{-1} v-\frac{j^{l}\left(\chi_{N}^{-1}\right)^{2}}{2}+W_{1}\left(\chi_{N}^{-1}\right)\right] d x \\
& =\int_{0}^{1}\left[\frac{1}{2} \chi_{N}^{-1} v-\frac{1}{2}+W_{1}\left(\chi_{N}^{-1}\right)\right] d x .
\end{aligned}
$$

On an interval $(a, b)$ where $\chi_{N}^{-1}$ is constant, say 1 ,

$$
\int_{a}^{b} \chi_{N}^{-1} v d x=\int_{a}^{b} v d x=\int_{a}^{b}\left[\chi_{N}^{-1}+\gamma^{2} v^{\prime \prime}\right] d x=(b-a)+\gamma^{2}\left[v^{\prime}(b)-v^{\prime}(a)\right] .
$$

This implies

$$
\begin{aligned}
I^{l}\left(\chi_{N}^{-1}\right) & =\gamma^{2}\left[-v^{\prime}\left(\xi_{1}\right)+v^{\prime}\left(\xi_{2}\right)+\ldots\right]+\xi_{1} W_{1}(-1)+\left(\xi_{2}-\xi_{1}\right) W_{1}(1)+\ldots \\
& =-N \gamma^{2} v^{\prime}\left(\xi_{1}\right)+\xi_{1} N W_{1}(-1)+\left(1-\xi_{1} N\right) W_{1}(1) .
\end{aligned}
$$

Therefore, for $N \geq 1$,

$$
\begin{aligned}
& \left(K+I^{l}\right)\left(\chi_{N}^{-1}\right) \\
& =c_{0} N-\gamma\left[1-\frac{1}{2} \int_{-1}^{1} f_{1}(r) d r\right] N \tanh \left(\frac{\xi_{1}}{\gamma}\right)-N \xi_{1} \int_{-1}^{1} f_{1}(r) d r+W_{1}(1) .
\end{aligned}
$$


Similar computation shows

$$
\left(K+I^{l}\right)\left(\chi_{N}^{1}\right)=\left(K+I^{l}\right)\left(\chi_{N}^{-1}\right), \quad N \geq 1 .
$$

Although (2.16) and (2.17) are obtained for $N \geq 1$, we can take $N$ in (2.16) and (2.17) to be a positive real number and send it to 0 . Then if $\int_{-1}^{1} f_{1}(r) d r>0$, (2.13) implies that $\xi_{1}$ approaches $\infty$ and $1 / N-\xi_{1}$ approaches a positive number. Thus $\xi_{1} N \rightarrow 1$, and $I^{l}\left(\chi_{N}^{ \pm 1}\right) \rightarrow W_{1}(-1)=\left(K+I^{l}\right)\left(\chi_{0}^{-1}\right)$. After analyzing the similar case $\int_{-1}^{1} f_{1}(r) d r<0$, we deduce

$$
\begin{aligned}
\lim _{N \rightarrow 0}\left(K+I^{l}\right)\left(\chi_{N}^{ \pm 1}\right) \\
=\left\{\begin{array}{l}
\left(K+I^{l}\right)\left(\chi_{0}^{-1}\right)<\left(K+I^{l}\right)\left(\chi_{0}^{1}\right), \text { if } \int_{-1}^{1} f_{1}(r) d r>0 \\
\left(K+I^{l}\right)\left(\chi_{0}^{-1}\right)=\left(K+I^{l}\right)\left(\chi_{0}^{1}\right), \text { if } \int_{-1}^{1} f_{1}(r) d r=0 \\
\left(K+I^{l}\right)\left(\chi_{0}^{1}\right)<\left(K+I^{l}\right)\left(\chi_{0}^{-1}\right), \text { if } \int_{-1}^{1} f_{1}(r) d r<0
\end{array}\right.
\end{aligned}
$$

On the other hand as $N \rightarrow \infty,\left(K+I^{l}\right)\left(\chi_{N}^{ \pm 1}\right)$ grows like $c_{0} N$. Moreover $\left(K+I^{l}\right)\left(\chi_{N}^{ \pm 1}\right)$ is indeed convex in $N$. This follows from carefully implicitly differentiating (2.13) and (2.16). It turns out

$$
\begin{aligned}
\frac{d\left(K+I^{l}\right)\left(\chi_{N}^{-1}\right)}{d N}= & c_{0}-\gamma(1-p) \tanh \left(\frac{\xi_{1}}{\gamma}\right)-2 p \xi_{1} \\
& +\frac{(1+p)^{2}}{2 N}\left[1-\left(\frac{1-p}{1+p}\right)^{2} \tanh ^{2}\left(\frac{\xi_{1}}{\gamma}\right)\right] \\
\frac{d^{2}\left(K+I^{l}\right)\left(\chi_{N}^{-1}\right)}{d N^{2}}= & \kappa \frac{\tanh \left(\frac{\xi_{1}}{\gamma}\right)\left[1-\tanh ^{2}\left(\frac{\xi_{1}}{\gamma}\right)\right]\left[1-\tanh ^{2}\left(\frac{1}{\gamma N}-\frac{\xi_{1}}{\gamma}\right)\right]_{2}}{2 \gamma N^{3}\left[1-\tanh \left(\frac{\xi_{1}}{\gamma}\right) \tanh \left(\frac{1}{\gamma N}-\frac{\xi_{1}}{\gamma}\right)\right]}
\end{aligned}
$$

where

$$
p=\frac{1}{2} \int_{-1}^{1} f_{1}(r) d r, \quad \kappa=(1-p)^{2}(1+p)>0 .
$$

From (2.20) we see $\frac{d^{2}\left(K+I^{l}\right)\left(\chi_{N}^{-1}\right)}{d N^{2}}>0$. The convexity of $\left(K+I^{l}\right)\left(\chi_{N}^{ \pm 1}\right)$ and the fact that $\lim _{N \rightarrow \infty} \frac{d I^{l}\left(\chi_{N}^{-1}\right)}{d N}=0$, imply $\frac{d I^{l}\left(\chi_{N}^{ - \pm 1}\right)}{d N}<0$, i.e. $I^{l}\left(\chi_{N}^{ \pm 1}\right)$ decreases in $N$.

We proceed to show that $\chi_{N}^{ \pm 1}$ is the global minimum of $I^{l}$ in $A_{N}^{ \pm 1}, N \geq 0$. Suppose this is not true. Since $I^{l}$ is bounded below in $A_{N}$, identified with the set $\left\{\left(\xi_{1}, \xi_{2}, \ldots, \xi_{N}\right) \in R^{N}: 0<\xi_{1}<\xi_{2}<\ldots<\xi_{N}<1\right\}$ and $\chi_{N}^{ \pm 1}$ is the only critical point, there exists a sequence $u_{n} \in A_{N}$ converging to a point $u$ on the boundary of $A_{N}$ with $\lim _{n \rightarrow \infty} I^{l}\left(u_{n}\right)=\inf _{u \in A_{N}^{ \pm 1}} I^{l}(u)$. This point $u$ is a member of $A_{N^{\prime}}^{ \pm 1}$ with $N^{\prime}<N$. If in $A_{N^{\prime}}^{ \pm 1}, \chi_{N^{\prime}}^{ \pm 1}$ is the global minimum of $I^{l}$, we find $I^{l}\left(\chi_{N}^{ \pm 1}\right) \geq I^{l}(u) \geq I^{l}\left(\chi_{N^{\prime}}^{ \pm 1}\right)$, which contradicts the fact that $I^{l}\left(\chi_{N}^{ \pm 1}\right)$ decreases in $N$. If $\chi_{N^{\prime}}^{ \pm 1}$ is not the global minimum of $I^{l}$ in $A_{N^{\prime}}^{ \pm 1}$, we repeat the same argument and end up in another $\chi_{N^{\prime \prime}}^{ \pm 1}$ with $N^{\prime \prime}<N^{\prime}$. This argument stops if we are in $A_{0}^{ \pm 1}$. There $\chi_{0}^{ \pm 1}$, the only element, is trivially the minimum.

We now summarize our results regarding the local minima of $K+I^{l}$ and $I_{\epsilon}$ in the next two theorems. 
Theorem 2.6 Let $J^{l}$ be defined by (2.11) and $f_{1}$ satisfies (2.15). The family of all local minima of $K+I^{l}$ is exactly $\left\{\chi_{N}^{ \pm 1}: N=0,1,2, \ldots\right\}$.

Proof. Take a function $u \in B_{\delta}\left(\chi_{N}^{-1}\right) \subset L^{2}(0,1), u \neq \chi_{N}^{-1}, \delta$ to be specified later. If $u \in A_{N}^{-1}$, then the assertion before this theorem implies $\left(K+I^{l}\right)(u)>$ $\left(K+I^{l}\right)\left(\chi_{N}^{-1}\right)$. If $u \in L^{2}(0,1) \backslash B V((0,1),\{-1,1\})$, then $\infty=\left(K+I^{l}\right)(u)>(K+$ $\left.I^{l}\right)\left(\chi_{N}^{-1}\right)$. We now consider the remaining case $u \in B V((0,1),\{-1,1\}) \backslash A_{N}^{-1}$. In this case $\frac{\|D u\|}{2}$, the number of jumps $u$ has, is either $\geq N+1$ or $\leq N-1$. The sub-case $\frac{\|D u\|}{2} \leq N-1$ does not happen by a property of BV-functions (see [16] Theorem 1, p. 172): If $u_{n} \rightarrow \chi_{N}^{-1}$ in $L^{1}$ then $\liminf _{n \rightarrow \infty}\left\|D u_{n}\right\|(0,1) \geq$ $\left\|D \chi_{N}^{-1}\right\|(0,1)=2 N$. So if we choose $\delta$ small, $\frac{\|D u\|(0,1)}{2}$ can not be less than or equal to $N-1$. In the sub-case $\frac{\|D u\|}{2} \geq N+1$,

$$
\begin{aligned}
\left(K+I^{l}\right)(u) & \geq c_{0}(N+1)+I^{l}(u) \\
& =\left(K+I^{l}\right)\left(\chi_{N}^{-1}\right)+I^{l}(u)-I^{l}\left(\chi_{N}^{-1}\right)+c_{0} \\
& >\left(K+I^{l}\right)\left(\chi_{N}^{-1}\right)
\end{aligned}
$$

if $\delta$ is chosen small enough, by the continuity of $I^{l}$.

Note that the conditions $\mathbf{H} \mathbf{4}$ and $\mathbf{H} \mathbf{8}$ are not needed here.

Theorem 2.7 Let $J^{l}$ be defined by (2.11) and $f_{1}$ satisfies (2.15). For each positive integer $\nu$ we can find $\delta>0$ such that

1. $\left\{B_{\delta}\left(\chi_{N}^{-1}\right), B_{\delta}\left(\chi_{N}^{1}\right): N=1,2, \ldots, \nu\right\}$ is a family of $2 \nu$ mutually disjoint open balls in $L^{2}(0,1)$;

2. for small $\epsilon$, there exist a local minimum $u_{N, \epsilon}^{-1}$ of $I_{\epsilon}$ in $B_{\delta}\left(\chi_{N}^{-1}\right)$ and a local minimum $u_{N, \epsilon}^{1}$ in $B_{\delta}\left(\chi_{N}^{1}\right)$, for $N=0,1, \ldots \nu$, satisfying $\lim _{\epsilon \rightarrow 0} \| u_{N, \epsilon}^{-1}-$ $\chi_{N}^{-1} \|_{2}=0$ and $\lim _{\epsilon \rightarrow 0}\left\|u_{N, \epsilon}^{1}-\chi_{N}^{1}\right\|_{2}=0$.

Proof. A consequence of Proposition 2.4 and Theorem 2.6.

Figure 3 shows two local minima of $I_{\epsilon}$ obtained by numerically minimizing $I_{\epsilon}$ in $B_{\delta}\left(\chi_{7}^{-1}\right)$ and $B_{\delta}\left(\chi_{8}^{-1}\right)$ in comparison with $\chi_{7}^{-1}$ and $\chi_{8}^{-1}$. In this calculation $\epsilon=0.005, \gamma=0.5, f_{1}(s)=-0.2 s$ and $J^{s}(r)=2.2 \exp (-|r|)$.

Next we study the global minima of $K+I^{l}$ and $I_{\epsilon}$. Because of the convexity (2.20), if we minimize $\left(K+I^{l}\right)\left(\chi_{N}^{ \pm 1}\right)$ among $N=0,1,2,3, \ldots$, for most $c_{0}$, it is achieved at one integer $N_{*}$, but for some countably many $c_{0}$, it is achieved at two consecutive integers $N_{*}$ and $N_{*}+1$. Also because of the specialty of $\left(K+I^{l}\right)\left(\chi_{0}^{ \pm 1}\right)$ shown in (2.18), we have altogether six possibilities.

Theorem 2.8 Let $J^{l}$ be defined by (2.11) and $f_{1}$ satisfies (2.15). Let $\mathcal{C}$ be the countable set of positive numbers such that $\left(K+I^{l}\right)\left(\chi_{N}^{ \pm 1}\right)$ is minimized at one non-negative integer $N_{*}$ if $c_{0} \notin \mathcal{C}$, and at two non-negative integers $N_{*}$ and $N_{*}+1$ if $c_{0} \in \mathcal{C}$.

1. If $c_{0} \notin \mathcal{C}, N_{*}=0$ and $\int_{-1}^{1} f_{1}(r) d r=0, K+I^{l}$ has two global minima $\chi_{0}^{ \pm 1}$. 

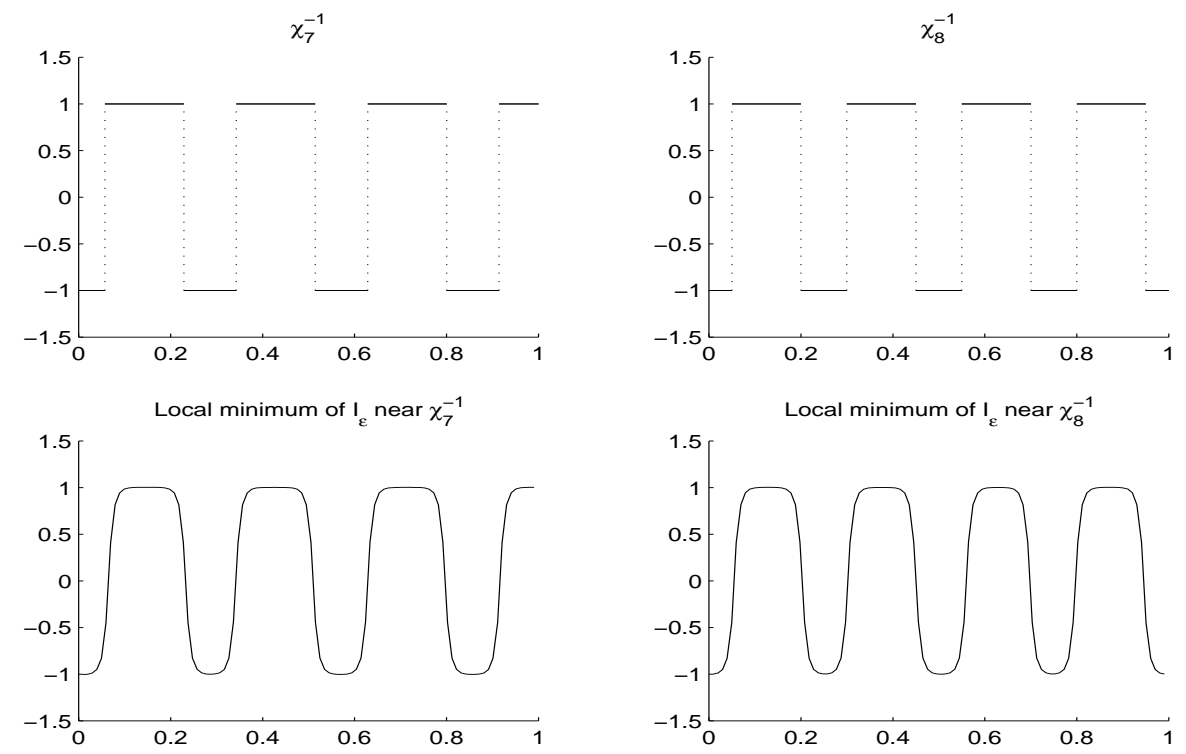

Figure 3: Local minima of $I_{\epsilon}$ near $\chi_{7}^{-1}$ and $\chi_{8}^{-1}$.

2. If $c_{0} \notin \mathcal{C}, N_{*}=0$ and $\int_{-1}^{1} f_{1}(r) d r \neq 0, K+I^{l}$ has one global minimum $\chi_{0}^{-1}$ if $\int_{-1}^{1} f_{1}(r) d r>0$, or $\chi_{0}^{1}$ if $\int_{-1}^{1} f_{1}(r) d r<0$.

3. If $c_{0} \notin \mathcal{C}$ and $N_{*} \geq 1, K+I^{l}$ has two global minima $\chi_{N_{*}}^{ \pm 1}$.

4. If $c_{0} \in \mathcal{C}, N_{*}=0$ and $\int_{-1}^{1} f_{1}(r) d r=0, K+I^{l}$ has four global minima $\chi_{0}^{ \pm 1}, \chi_{1}^{ \pm 1}$.

5. If $c_{0} \in \mathcal{C}, N_{*}=0$ and $\int_{-1}^{1} f_{1}(r) d r \neq 0, K+I^{l}$ has three global minima $\chi_{0}^{-1}, \chi_{1}^{ \pm 1}$ if $\int_{-1}^{1} f_{1}(r) d r>0$, or $\chi_{0}^{1}, \chi_{1}^{ \pm 1}$ if $\int_{-1}^{1} f_{1}(r) d r<0$.

6. If $c_{0} \in \mathcal{C}, N_{*} \geq 1, K+I^{l}$ has four global minima $\chi_{N_{*}}^{ \pm 1}, \chi_{N_{*}+1}^{ \pm 1}$.

Again the conditions $\mathbf{H 4}$ and $\mathbf{H 8}$ are not needed in this theorem.

Theorem 2.9 Let $J^{l}$ be defined by (2.11), $f_{1}$ satisfy (2.15). $\mathcal{C}$ and $N_{*}$ are the same as in Theorem 2.8. Let $u_{\epsilon}$ be a global minimum of $I_{\epsilon}$.

1. If $c_{0} \notin \mathcal{C}, N_{*}=0$ and $\int_{-1}^{1} f_{1}(r) d r=0$, then for every $\delta>0$ and small $\epsilon$, $u_{\epsilon}$ is in $B_{\delta}\left(\chi_{0}^{-1}\right) \cup B_{\delta}\left(\chi_{0}^{1}\right)$.

2. If $c_{0} \notin \mathcal{C}, N_{*}=0$ and $\int_{-1}^{1} f_{1}(r) d r \neq 0$, then for every $\delta>0$ and small $\epsilon$, $u_{\epsilon}$ is in $B_{\delta}\left(\chi_{0}^{-1}\right)$ if $\int_{-1}^{1} f_{1}(r) d r>0$, or in $B_{\delta}\left(\chi_{0}^{1}\right)$ if $\int_{-1}^{1} f_{1}(r) d r<0$. 
3. If $c_{0} \notin \mathcal{C}$ and $N_{*} \geq 1$, then for every $\delta>0$ and small $\epsilon, u_{\epsilon}$ is in $B_{\delta}\left(\chi_{N_{*}}^{-1}\right) \cup$ $B_{\delta}\left(\chi_{N_{*}}^{1}\right)$.

4. If $c_{0} \in \mathcal{C}, N_{*}=0$ and $\int_{-1}^{1} f_{1}(r) d r=0$, then for every $\delta>0$ and small $\epsilon$, $u_{\epsilon}$ is in $B_{\delta}\left(\chi_{0}^{-1}\right) \cup B_{\delta}\left(\chi_{0}^{1}\right) \cup B_{\delta}\left(\chi_{1}^{-1}\right) \cup B_{\delta}\left(\chi_{1}^{1}\right)$.

5. If $c_{0} \in \mathcal{C}, N_{*}=0$ and $\int_{-1}^{1} f_{1}(r) d r \neq 0$, then for every $\delta>0$ and small $\epsilon, u_{\epsilon}$ is in $B_{\delta}\left(\chi_{0}^{-1}\right) \cup B_{\delta}\left(\chi_{1}^{-1}\right) \cup B_{\delta}\left(\chi_{1}^{1}\right)$ if $\int_{-1}^{1} f_{1}(r) d r>0$, or $B_{\delta}\left(\chi_{0}^{1}\right) \cup$ $B_{\delta}\left(\chi_{1}^{-1}\right) \cup B_{\delta}\left(\chi_{1}^{1}\right)$ if $\int_{-1}^{1} f_{1}(r) d r<0$.

6. If $c_{0} \in \mathcal{C}, N_{*} \geq 1$, then for every $\delta>0$ and small $\epsilon, u_{\epsilon}$ is in $B_{\delta}\left(\chi_{N_{*}}^{-1}\right) \cup$ $B_{\delta}\left(\chi_{N_{*}}^{1}\right) \cup B_{\delta}\left(\chi_{N_{*}+1}^{-1}\right) \cup B_{\delta}\left(\chi_{N_{*}+1}^{1}\right)$.

Proof. A consequence of Proposition 2.5 and Theorem 2.8.

Remark 2.10 In the case $\int_{-1}^{1} f_{1}(s) d s=0$, we simply have $\xi_{1}=\frac{\xi_{2}-\xi_{1}}{2}=\frac{1}{2 N}$,

$$
\begin{aligned}
\left(K+I^{l}\right)\left(\chi_{N}^{ \pm 1}\right) & =c_{0} N-\gamma N \tanh \left(\frac{1}{2 \gamma N}\right)+W(1), \\
\frac{d^{2}\left(K+I^{l}\right)\left(\chi_{N}^{ \pm 1}\right)}{d N^{2}} & =\frac{\tanh \left(\frac{1}{2 \gamma N}\right)\left[1-\tanh ^{2}\left(\frac{1}{2 \gamma N}\right)\right]}{2 \gamma N^{3}} .
\end{aligned}
$$

Remark 2.11 After (2.9), we can find the matrix $\left[\frac{\partial^{2} I^{l}}{\partial \xi_{i} \partial \xi_{j}}\right]$,

$$
\frac{\partial^{2} I^{l}}{\partial \xi_{i} \partial \xi_{j}}=4 \times\left\{\begin{array}{ll}
J^{l}\left(\xi_{i}, \xi_{i}\right)+\sum_{k=1}^{N}(-1)^{i+k+1} G\left(\xi_{i}, \xi_{k}\right) & \text { if } i=j \\
(-1)^{i+j} J^{l}\left(\xi_{i}, \xi_{j}\right) & \text { if } i \neq j
\end{array},\right.
$$

where $G$ is the Green function of the equation $-\gamma^{2} w+w=g$ with the Dirichlet boundary condition $w(0)=w(1)=0$.

This matrix will be compared to (3.9) of Section 3, although we did not use it in the study of local minima $\chi_{N}^{ \pm 1}$.

Remark 2.12 We expect that the results presented here can be extended to the $\mathrm{J}^{l}$ 's that are Green's functions of general ODEs of the divergence form

$$
-\left(p(x) v^{\prime}(x)\right)^{\prime}+q(x) v(x)=u, a_{0} v(0)+b_{0} v^{\prime}(0)=a_{1} v(1)+b_{1} v^{\prime}(1)=0 .
$$

In this case we can not expect the good spacing result (2.12). The location of interfaces will be affected by $p$ and $q$. 


\section{The matched asymptotics approach}

In this section we study solutions on the real line. Since there is no boundary, $J^{l}$ is taken to be even and translationally invariant: $J(x, y)=J(|x-y|)$. We again separate the long range and short range effects, more precisely, we assume that the short range effect is localized and the long range effect is small:

$$
J(r)=J_{\epsilon}^{s}(r)-\epsilon J^{l}(r)=\frac{1}{\epsilon} J^{s}\left(\frac{r}{\epsilon}\right)-\epsilon J^{l}(r) .
$$

In order to see the motion of interfaces, we need to consider (1.13) on a slower time scale. Similar to the Allen-Cahn equation we re-scale the time variable by $\epsilon^{2}$ and consider

$$
\epsilon^{2} u_{t}=J_{\epsilon}^{s} * u-\epsilon J^{l} * u-j u-f(u),
$$

where $*$ is defined by $J * u(x)=\int_{R} J(x-y) u(y) d y$, and $j=\int_{R} J(r) d r$. About $J^{s}$ and $J^{l}$ we assume $J^{s} \geq 0$ and $j^{l}=\int_{R} J^{l}(r) d r>0$. It will be important below that $J^{l}$ is allowed to change sign. About $f$ we assume that $f(u)=f_{0}(u)+\epsilon f_{1}(u)$, $f_{0}$ is bistable with zeros at $-1, a$ and $1, f_{0}$ is balanced, i.e., $\int_{-1}^{1} f_{0}(u) d u=0$ and $j^{s}+f_{0}^{\prime}>0$, where $j^{s}=\int_{R} J^{s}(r) d r$. In addition, we assume that for some constant $C$ and all $x$,

$$
J^{s}(x)\left|x^{3}\right| \leq C .
$$

The analysis that follows uses a matched asymptotics method, in the style of [17] or [20]. Though our discussion is formal, we believe it can be made rigorous by e.g., an appropriate use of the implicit function theorem technique.

We consider families $u(x, t ; \epsilon)$ of solutions with the "layer" property, where $x \in R$, defined for all small $\epsilon>0$. We suppose that $u$ has "layers" at a finite sequence $\xi_{1}(t), \ldots, \xi_{N}(t)$. For all $i$, we assume $u\left(\xi_{i}(t), t ; \epsilon\right)=0$. Let $\Gamma(t) \equiv \cup \xi_{i}(t)$. At locations bounded away from $\Gamma(t), u$ can be described by an outer expansion

$$
u(x, t ; \epsilon)=u_{0}(x, t)+\epsilon u_{1}(x, t)+\ldots .
$$

Changing variables, we may express the convolution $J_{\epsilon}^{s} * u$ in the form

$$
J_{\epsilon}^{s} * u(x, t)=\int_{R} J^{s}(y) u(x+\epsilon y, t) d y=j^{s} u_{0}(x, t)+\epsilon j^{s} u_{1}(x, t)+O\left(\epsilon^{2}\right) .
$$

Substituting the last two equations into (3.2), we obtain

$$
0=-f_{0}\left(u_{0}\right)-\epsilon\left[f_{0}^{\prime}\left(u_{0}\right) u_{1}+f_{1}\left(u_{0}\right)+J^{l} * u_{0}-j^{l} u_{0}\right]+O\left(\epsilon^{2}\right) .
$$

Therefore $f_{0}\left(u_{0}\right)=0$. For $u_{0}$ we select the outermost zeros of $f_{0}$, i.e.,

$$
u_{0}(x, t)= \pm \begin{cases}-1, & x \in I_{N}^{-1}=\left(-\infty, \xi_{1}(t)\right) \cup\left(\xi_{2}(t), \xi_{3}(t)\right) \ldots\left(\xi_{N}(t), \infty\right), \\ 1, & x \in R \backslash I_{N}^{-1},\end{cases}
$$

if $N$ is even, and

$$
u_{0}(x, t)= \pm \begin{cases}-1, & x \in I_{N}^{-1}=\left(-\infty, \xi_{1}(t)\right) \cup\left(\xi_{2}(t), \xi_{3}(t)\right) \ldots\left(\xi_{N-1}(t), \xi_{N}(t)\right), \\ 1, & x \in R \backslash I_{N}^{-1}\end{cases}
$$


if $N$ is odd. $u_{1}$, etc., can in turn be determined successively from (3.4) and its extensions.

The representation of $u$ near $\Gamma(t)$ is as follows. Let $z=\frac{x-\xi_{i}(t)}{\epsilon}$ be the stretched variable. We assume that there exists a neighborhood $N^{\epsilon}(t)$ of $\Gamma(t)$, and a small number $r>0$, such that in any ball of radius $r$, centered at any point $x$ in $N(t)$, both $u(x, t)$ and $v(x, t) \equiv J^{l} * u(x, t)$ can be expressed in the form:

$$
u(x, t ; \epsilon)=U^{i}(z, t ; \epsilon) ; v(x, t ; \epsilon)=V^{i}(z, t ; \epsilon) .
$$

Let $B(x, t)$ denote the ball of that radius centered at $x$. Note that by (3.3), for $y \notin B(x, t), J_{\epsilon}^{s}(x-y) \leq \frac{C \epsilon^{2}}{|x-y|^{3}}$, so that if the quantity $J_{\epsilon}^{s} * u(x, t)$ is replaced by the integral only over $B(x, t)$, we incur an error only of the order $O\left(\epsilon^{2}\right)$. Thus we have

$$
J_{\epsilon}^{s} * u(x, t)=\int_{B(x, t)} \frac{1}{\epsilon} J^{s}\left(\frac{x-y}{\epsilon}\right) u(y, t) d y+O\left(\epsilon^{2}\right)=J^{s} * U^{i}(z, t)+O\left(\epsilon^{2}\right)
$$

Since

$$
\frac{\partial U^{i}}{\partial t}=-\frac{1}{\epsilon} U_{z}^{i} \dot{\xi}_{i}+O(1)
$$

the inner solutions are given by

$$
-\epsilon U_{z}^{i} \dot{\xi}_{i}=J^{s} * U^{i}-j^{s} U^{i}-f\left(U^{i}\right)-\epsilon\left(V^{i}-j^{l} U^{i}\right)+O\left(\epsilon^{2}\right) .
$$

We assume that each $U^{i}$ and $V^{i}$ can be described by the expansions

$$
\begin{aligned}
& U^{i}(z, t)=U_{0}^{i}(z, t)+\epsilon U_{1}^{i}(z, t)+\ldots, \\
& V^{i}(z, t)=V_{0}^{i}(z, t)+\epsilon V_{1}^{i}(z, t)+\ldots,
\end{aligned}
$$

Substituting these into (3.5), to lowest order we obtain

$$
J^{s} * U_{0}^{i}-j^{s} U_{0}^{i}-f_{0}\left(U_{0}^{i}\right)=0 .
$$

We now recall from [6] that if $j^{s}+f_{0}^{\prime}>0$ and $f_{0}$ is balanced, then this equation has two stationary and stable front solutions $U_{0}^{+}$and $U_{0}^{-}$, which are differentiable and (suppressing dependence on $t$ ) such that $U_{0}^{+}( \pm \infty)= \pm 1$ and $U_{0}^{-}( \pm \infty)=\mp 1$ (it is also easy to see that if $U_{0}^{+}(0)=U_{0}^{-}(0)=0$ then $\left.U_{0}^{+}(z)=U_{0}^{-}(-z)\right)$. These solutions will match the behavior of the outer solutions $u_{0}(x)$ and $v_{0}(x)$ near the interfaces $\xi_{i}(t)$, which implies

$$
U_{0}^{i}( \pm \infty, t)=u_{0}\left(\xi_{i}(t) \pm, t\right),
$$

where $U_{0}^{i}$ denotes either $U_{0}^{+}$or $U_{0}^{-}$. The location and number of possible interfaces is determined from the equation for the $\epsilon$ coefficient in (3.5):

$$
L U_{1}^{i} \equiv J^{s} * U_{1}^{i}-j^{s} U_{1}^{i}-f_{0}^{\prime}\left(U_{0}^{i}\right) U_{1}^{i}=-U_{0}^{i^{\prime}} \dot{\xi}_{i}+f_{1}\left(U_{0}^{i}\right)+V_{0}^{i}-j^{l} U_{0}^{i}+O(\epsilon) .
$$


To analyze this equation, first note that

$$
\begin{aligned}
J^{l} * u(z, t) & =\int_{R} J^{l}\left(\xi_{i}(t)-y\right) u(y+\epsilon z, t) d y \\
& =\int_{R} J^{l}\left(\xi_{i}(t)-y\right)\left(u_{0}(y, t)+O(\epsilon)\right) d y \\
& =J^{l} * u_{0}\left(\xi_{i}(t)\right)+O(\epsilon)
\end{aligned}
$$

thus $V_{0}^{i}(z, t)=J^{l} * u_{0}\left(\xi_{i}(t)\right)$ for all $z$.

Also, recall from [6] that (3.6) is solvable (in $L_{2}$ ) if and only if $L U_{1}^{i}$ is orthogonal to the simple eigenfunction of $L$, i.e., to $U_{0}^{i^{\prime}}$. This condition applied to (3.6) yields

$$
\int_{R}\left[-U_{0}^{i^{\prime}} \dot{\xi}_{i}+f_{1}\left(U_{0}^{i}\right)-j^{l} U_{0}^{i}+J^{l} * u_{0}\left(\xi_{i}\right)+O(\epsilon)\right] U_{0}^{i^{\prime}}=0 .
$$

The leading order equation. To the lowest order, we deduce a system of autonomous, nonlinear ODE's:

$$
\dot{\xi}_{i}(t)= \pm \frac{2}{\left\|U_{0}^{\prime}\right\|_{2}^{2}}\left[J^{l} * u_{0}\left(\xi_{i}(t)\right)+\frac{1}{2} \int_{-1}^{1} f_{1}(r) d r\right],
$$

where the signs + and - correspond to $U_{0}^{+}$and $U_{0}^{-}$, respectively. Existence of "layer" solutions is determined from the equilibria of (3.7). The layers $\xi_{i}$ thus satisfy the system of algebraic equations:

$$
J^{l} * u_{0}\left(\xi_{i}\right)=-\frac{1}{2} \int_{-1}^{1} f_{1}(r) d r .
$$

Not surprisingly this is the whole interval version of (2.10) in Section 2.

Let $\hat{u}(x ; \epsilon)$ denote a possible stationary solution of $(3.2)$. We formally assume that the linearization of the right side of (3.7) about the layers of $\hat{u}(x ; \epsilon)$ determines the stability of $\hat{u}(x ; \epsilon)$. Let $N$ be the number of layers. Then this linearization leads to the $N \times N$ matrix $\frac{2}{\left\|U_{0}^{\prime}\right\|_{2}^{2}}\left[a_{i j}\right]$, where

$$
a_{i j}=\left\{\begin{array}{ll}
\sum_{k=1, k \neq i}^{N}(-1)^{i+k} J^{l}\left(\xi_{i}-\xi_{k}\right), & i=j \\
(-1)^{i+j+1} J^{l}\left(\xi_{i}-\xi_{j}\right), & i \neq j
\end{array} .\right.
$$

Note that the negative of this matrix is the real line version of the matrix in Remark 2.11. Since $\hat{u}$ is translationally invariant, one of the eigenvalues of this matrix is zero. We make the formal assumption that the stability of $\hat{u}$ follows if the other eigenvalues of $\left[a_{i j}\right]$ are negative.

We first focus our attention on solutions of heteroclinic and homoclinic type, with 1, 2 or 3 layers. We remark here that (3.8) is not always solvable, so solutions with more than 3 layers may not exist.

1-layer solutions. Here, (3.8) becomes:

$$
\mp \int_{-\infty}^{\xi_{1}} J^{l}\left(y-\xi_{1}\right) d y \pm \int_{\xi_{1}}^{\infty} J^{l}\left(y-\xi_{1}\right) d y=-\frac{1}{2} \int_{-1}^{1} f_{1}(r) d r .
$$


Since $J^{l}$ is even, the left side is zero. Then the right side is also zero, and the only two solutions are given by the formulas: $u_{0}(x)= \pm\left[H\left(x-\xi_{1}\right)-H\left(-x-\xi_{1}\right)\right]$, where $H$ is the Heaviside function. From the discussion above, they are stable.

2-layer solutions. Here, we look for solutions of the form:

$$
u_{0}(x)= \pm\left\{\begin{array}{rl}
-1 & x<\xi_{1} \text { and } x>\xi_{2} \\
1 & \xi_{1}<x<\xi_{2}
\end{array}\right.
$$

The two equations in (3.8) for $\xi_{1}$ can be reduced to a single one:

$$
\pm \int_{\xi_{1}}^{\xi_{2}} J^{l}(y) d y=\frac{j^{l}}{2}-\frac{1}{4} \int_{-1}^{1} f_{1}(r) d r .
$$

The number of solutions and their stability depends on $j^{l}, \int_{-1}^{1} f_{1}$ and also on the number of sign changes of $J^{l}$. Assuming that $J^{l}$ is positive and $J^{l}(r)$ is strictly decreasing for $r>0$, there exists a solution $u_{0}$ of the form (3.10), with a unique difference $\xi_{2}-\xi_{1}$, under the additional condition

$$
0< \pm \int_{-1}^{1} f_{1}(r) d r<2 j^{l}
$$

Note that a similar condition, (2.15), has appeared in Section 2. The matrix given by (3.9) is

$$
\left[\begin{array}{rr}
-J^{l}(d) & J^{l}(d) \\
J^{l}(d) & J^{l}(d)
\end{array}\right]
$$

where $d=\xi_{2}-\xi_{1}$. Its two eigenvalues are: $\lambda_{1}=0$ and $\lambda_{2}=-2 J^{l}(d)$, thus the homoclinic is stable.

3-layer solutions. Here, we look for solutions of the form

$$
u_{0}(x)= \pm\left\{\begin{array}{rl}
-1 & x<\xi_{1} \text { and } \xi_{2}<x<\xi_{3} \\
1 & \xi_{1}<x<\xi_{2} \text { and } x>\xi_{3}
\end{array}\right.
$$

(3.8) has such solutions only for $\int_{-1}^{1} f_{1}=0$. The equations in (3.8) can be reduced to the system

$$
\left\{\begin{array}{l}
\left(\int_{\xi_{1}}^{\xi_{2}}+\int_{\xi_{3}}^{\infty}\right) J^{l}\left(y-\xi_{1}\right) d y=\frac{j^{l}}{2} \\
\left(\int_{\xi_{1}}^{\xi_{2}}+\int_{\xi_{3}}^{\infty}\right) J^{l}\left(y-\xi_{3}\right) d y=\frac{j^{l}}{2}
\end{array}\right.
$$

or, using the fact that $J^{l}$ is even, to

$$
\left\{\begin{array}{l}
\int_{\xi_{3}}^{\xi_{3}} J^{l}(y) d y=0 \\
\int_{\xi_{3}-\xi_{1}}^{\xi_{3}-\xi_{2}} J^{l}(y) d y=0
\end{array}\right.
$$

Obviously, there are no solutions if $J^{l}>0$. For simplicity, we assume that there is some $x_{0}$ such that $J^{l}(x)>0$ for $|x|<x_{0}$ and $J^{l}(x)<0$ for $|x|>x_{0}$ Then there exists a solution $\left(\xi_{1}, \xi_{2}, \xi_{3}\right)$, moreover it satisfies: $J^{l}\left(\xi_{2}-\xi_{1}\right)>0$, 
$J^{l}\left(\xi_{3}-\xi_{1}\right)<0$ and has unique differences $\xi_{3}-\xi_{2}=\xi_{2}-\xi_{1}$. The matrix given by $(3.9)$ is

$$
\left[\begin{array}{crc}
-J^{l}(d)+J^{l}(2 d) & J^{l}(d) & -J^{l}(2 d) \\
J^{l}(d) & -2 J^{l}(d) & J^{l}(d) \\
-J^{l}(2 d) & J^{l}(d) & -J^{l}(d)+J^{l}(2 d)
\end{array}\right],
$$

where $d=\xi_{2}-\xi_{1}$. Instead of calculating the eigenvalues of this matrix explicitly, we look at the determinants of nested principal minors. The first is negative, the second positive, the third zero, so there is one zero and two negative eigenvalues. The heteroclinic solution is stable.

Traveling waves. An interesting by-product of having both a 1-layer and 3-layer stable solutions is that we can give an example of equation (3.2) which admits two (stable) traveling waves with different velocities.

The speed of traveling waves is faster than the speed of the interfaces earlier. We return to the original time scale of (1.13).

Assume that $\hat{u}(x ; \epsilon)$ is a stable heteroclinic solution of (3.2), and fix $\epsilon$. Consider the $\delta$-perturbed equation

$$
u_{t}=J_{\epsilon}^{s} * u-\epsilon J^{l} * u-j u-f_{0}(u)-\delta f_{1}(u)
$$

and assume that

$$
\int_{-1}^{1} f_{1}(r) d r \neq 0
$$

With $\epsilon$ fixed, we seek $\delta$-dependent slow traveling waves of (3.11) of the form $u(x-\delta c t ; \delta)$. We assume $u$ is defined for all small $\delta>0$ and can be expanded into formal power series:

$$
u(x-\delta c t ; \delta)=u_{0}(x)+\delta\left[u_{1}(x)-c t u_{0}^{\prime}(x)\right]+\ldots .
$$

Substituting this expansion into (3.11), we find

$$
\begin{gathered}
-c \delta u_{0}^{\prime}=J_{\epsilon}^{s} * u_{0}-\epsilon J^{l} * u_{0}-j u_{0}-f_{0}\left(u_{0}\right) \\
+\delta\left(J_{\epsilon}^{s} * u_{1}-\epsilon J^{l} * u_{1}-j u_{1}-f_{0}^{\prime}\left(u_{0}\right) u_{1}-f_{1}\left(u_{0}\right)\right)+O\left(\delta^{2}\right) .
\end{gathered}
$$

To the leading order, we get a stationary version of (3.2), thus we take

$$
u_{0}(x)=\hat{u}(x ; \epsilon) .
$$

Setting the $\delta$ coefficient of (3.12) to zero, we deduce

$$
L u_{1} \equiv J_{\epsilon}^{s} * u_{1}-\epsilon J^{l} * u_{1}-j u_{1}-f_{0}^{\prime}\left(u_{0}\right) u_{1}=-c u_{0}^{\prime}+f_{1}\left(u_{0}\right) .
$$

This equation is solvable if and only if $L u_{1}$ is orthogonal to the simple eigenfunction of $L$, i.e., to $u_{0}^{\prime}$. This condition yields

$$
c=\frac{\int_{-1}^{1} f_{1}(r) d r}{\left\|u_{0}^{\prime}\right\|_{2}^{2}} .
$$


It can be formally calculated that $\left\|u_{0}^{\prime}\right\|_{2}^{2}$ is proportional to the number of layers of $u_{0}$, thus we get two traveling waves, with the "3-layer" one being the slower one. Moreover, since (3.11) is a regularly perturbed version of (3.2), both waves are stable.

The matched asymptotics method presented in this section can be applied to (1.13) on a bounded interval. Then the motion of interfaces is governed by (3.7) with $J^{l} * u_{0}$ replaced by $J^{l}\left[u_{0}\right]$. All the rigorous results presented in Section 2 can be discovered using this formal method.

We may also find periodic solutions on the entire line. They are similar to the ones found in Section 2, extended periodically to the line. In general periodic equilibria of (1.13) have lower average energy per unit length than the heteroclinic, homoclinic, and traveling wave solutions found in this section. Periodic solutions are more preferred configurations energetically.

Our methods can also be used to other nonlocal equations, e.g., to the models discussed in [15], [14] or [11].

\section{Conclusions}

We studied a model with a nonlocal constitutive relation. It can be applied to complex binary materials like elastic crystals undergoing martensitic phase transitions and diblock copolymers below critical temperatures showing microphase separation.

We first formulated the underline free energy structure of the model and studied it on a finite interval. We found an infinite family of local minimizers of the energy for a particular form of the nonlocality. Each of them has a number of interfaces located periodically in the domain. With these minimizers we may model materials exhibiting periodic microstructures.

Next we considered an evolution equation of the nonlocal model. We derived the law governing the motion of interfaces. We constructed various stable equilibria on the entire line, asymptotically equal to one of the two constant stable states. This asymptotic behavior prevents those solutions from having a large number of layers. We also constructed traveling fronts with shapes similar to some of the equilibria. We conjecture that those solutions can appear only in the early stages of phase separation. Generically, the energetically preferred configurations are periodic.

The reader may compare our results with those for the Allen-Cahn equation (1.16). There, on a finite interval, only constant solutions are stable. Solutions with multiple interfaces are metastable, in the sense that the unstable eigenvalues of the linearized operators are exponentially small, see, e.g., [10]. On the entire line, the only stable solutions are one layer fronts.

\section{References}

[1] G. Alberti And G. Bellettini, A non-local anisotropic model for phase 
transitions: asymptotic behavior of rescaled enegries, Euro. J. Appl. Math., 9 (1998), 261-284.

[2] S. Allen and J.W. Cahn, A microscopic theory for antiphase boundary motion and its application to antiphase domain coarsening, Acta Metall., 27 (1979), 1084-1095.

[3] P.W. Bates and A. Chmaj, An integrodifferential model for phase transitions: stationary solutions in higher space dimensions, J. Stat. Phys., 95 (1999), 1119-1139.

[4] P.W. Bates and A. Chmaj, On a discrete convolution model for phase transitions, Arch. Rat. Mech. Anal., to appear.

[5] P.W. Bates, P.C. Fife, R. Gardner and C.K.R.T. Jones, The existence of traveling wave solutions of a generalized phase-field model, SIAM J. Math. Anal., 28 (1997), 60-93.

[6] P.W. Bates, P.C. Fife, X. Ren, and X. Wang, Traveling waves in a convolution model for phase transitions, Arch. Rat. Mech. Anal., 138 (1997) 105-136.

[7] P.W. Bates and X. Ren, Heteroclinic orbits for a higher order phase transition problem, Euro J. Appl. Math., 8 (1997), 149-163.

[8] P.W. Bates And X. Ren, Transition layer solutions of a higher order equation in an infinite tube, Comm. Partial Diff. Eq., 21 (1996), 195-220.

[9] W.L. Bragg and E.J. Williams, Effect of thermal agitation on atomic arrangement in alloys, Proc. Roy. Soc. London Ser. A, 145 (1934), 699-730.

[10] J. Carr and R.L. Pego, Metastable patterns in solutions of $u_{t}=\epsilon^{2} u_{x x}-$ $f(u)$, Comm. Pure Appl. Math,, 42 (1989), 523-576.

[11] X. Chen, Existence, uniqueness, and asymptotic stability of traveling waves in nonlocal evolution equations, Adv. Diff. Eq. 2 (1997), 125-160.

[12] A. Chmaj and X. Ren, Homoclinic solutions of an integral equation: existence and stability, J. Diff. Eq., 155 (1999), 17-43.

[13] G. DAL Maso, An Introduction to $\Gamma$-convergence, Progress in Nonlinear Diff. Eqn. and Appl., Birkhäuser, Boston 1993.

[14] A. De Masi, T. Gobron And E. Presutti, Traveling fronts in non local evolution equations, Arch. Rat. Mech. Anal. 132 (1995), 143-205.

[15] G.B. Ermentrout And J.B. McLeod, Existence and uniqueness of traveling waves for a neural network, Proc. Roy. Soc. Edin. 123A (1993), 461478. 
[16] L.C. Evans And R.F. Gariepy, Measure theory and fine properties of functions, CRC Press, Boca Raton New York London Tokyo, 1992.

[17] P.C. Fife, Models for phase separation and their mathematics, in Nonlinear Partial Differential Equations with Applications to Patterns, Waves and Interfaces (M. Mimura and T. Nishida, eds.), 183-212, Proc. Conf. on Nonlinear Partial Differential Equations, Kyoto (1992).

[18] P.C. Fife and M. KowalczyK, A class of pattern-forming systems, J. Nonlinear Science, 9 (1999), 641-669.

[19] R.M. Hornreich, M. Luban and S. Shtrikman, Critical behavior at the onset of $\vec{k}$-space instability on the $\lambda$-line, Phys. Rev. Lett. 35 (1975), 1678-1681.

[20] P.C. Fife And X. WANG, A convolution model for interfacial motion: the generation and propagation of internal layers in higher space dimensions, Adv. Diff. Eq. 3 (1998), 85-110.

[21] R. Kohn and P. Sternberg, Local minimisers and singular perturbations, Proc. Royal Soc. Edin., 111A (1989), 69-84.

[22] T. Ohta and K. KaWasaki, Equilibrium morphology of block polymer melts, Macromolecules, 19, 2621-2632, 1986.

[23] X. Ren and L. Truskinovsky, Finite scale microstructures in nonlocal elasticity, J. Elasticity, to appear.

[24] X. Ren and J. Wei, On the multiplicity of solutions of two nonlocal variational problems, SIAM J. Math. Anal., to appear.

[25] J.B. Swift And P.C. Hohenberg, Hydrodynamic fluctuations at the convective instability, Phys. Rev. A 15 (1977), 319-382.

[26] C. J. Thompson, Classical equilibrium statistical mechanics, Oxford University Press (1988). 